\title{
Managing Federations and Cooperative Management
}

\author{
Brendan Jennings $・$ Kevin Feeney $•$ Joel J. Fleck II
}

Received: 26 February 2014/ Accepted: 14 March 2014/Published online: 21 March 2014

(C) Springer Science+Business Media New York 2014

\section{Motivation}

Welcome to this special issue of the Journal of Network and Systems Management, which focusses on Management of Federations and Cooperative Management.

Over many years the research community has been actively investigating how network management systems can be evolved to achieve the flexibility and adaptability required to efficiently operate increasingly complex, heterogeneous and interconnected networks. Notable amongst these efforts has been the vision of autonomic networking [3,7] in which a network has the ability to self-govern its behaviour within the constraints of human-specified business goals. Autonomic networking promises a much more flexible approach to management that seeks to both allow systems to automatically adapt offered services or resources in response to user or environmental changes and to reduce operational expenditure for network operators.

Whilst significant progress has been made, this work has mainly focused on management in the context of single, well-defined network domains. Relatively little work has been done on how management systems can be federated across management domains to provide end-to-end management of communications services [6]. Current network management systems do perform some coordination

B. Jennings $(\bowtie)$

TSSG, Waterford Institute of Technology, Waterford, Ireland

e-mail: bjennings@ieee.org

K. Feeney

KDEG, Trinity College Dublin, Dublin, Ireland

e-mail: kevin.feeney@cs.tcd.ie

J. J. Fleck II

Hewlett Packard Inc., Palo Alto, CA, USA

e-mail: joel.fleck@hp.com 
across domains, but this is limited in scope to a small number of pre-defined information exchanges. This special issue addresses how management systems should be designed to incorporate capabilities supporting the negotiation and lifecycle management of federations involving networks, service providers and service consumers.

There are many management approaches and technologies than can be applied across organisational boundaries. These range from algorithmic approaches which adapt to the observed behaviour of third-party systems, based on game-theoretic approaches or other predictive models, to explicit organisational federations which adopt coherent solutions and management models to facilitate interoperability among multiple independent organisations. In particular, recent years have seen a wealth of research and technology developments into federated and cooperatively managed systems:

- Research in policy based management systems and Service Level Agreement (SLA) frameworks increasingly addresses sophisticated federated, inter-organisational relationships, particularly as they relate to SLA usage in cloud computing environments;

- Cooperative management research investigates how algorithmic approaches can facilitate non-destructive cross-domain inter-operation in the absence of explicit coordination;

- Grid and cloud computing research is concerned with the problem of making heterogeneous distributed computing resources available in flexible ways (including across administrative boundaries) to provide infrastructure as a service;

- Federated security research investigates the problems of providing trusted mechanisms on top of heterogeneous distributed security systems;

- Service Oriented Architectures and Internet mash-ups are increasingly enabling applications to be constructed based upon a diversity of components, drawn from multiple organisations systems;

- Data federation research investigates how data from heterogeneous sources can be aggregated into a normalised view that can be seamlessly leveraged by multiple applications;

- Research into lifecycles used to model multi-role Cloud Computing environments is investigating interoperation and federation among multiple artifact lifecycles;

- Model Driven Engineering (MDE) research is developing techniques that can be used to facilitate the specification, deployment and management of federations between heterogeneous and independent organisations.

This work has led to a growing realisation of the range of significant, complex issues which must be addressed in all technologies and applications that involve explicity or implicitly federated organisations. In an effort to identify and address these issues, a group of network and service management researchers organised the IFIP/IEEE International Workshop on Managing Federations and Cooperative Management in $2011[4,5]$. One of the outputs from this workshop was the proposal 
of this JNSM special issue, which seeks to provide an in-depth view of significant research outputs on the management of federations.

\section{Article summaries}

For this special issue we have selected four articles, addressing different challenges relating to management of federations.

The first article, "Managed Semantic Interoperability for Federations," by Brennan et al., recognises that achieving semantic interoperability between components is a pre-requisite to federating IT systems. The authors address dynamic federations in which autonomous members with heterogeneous data or service description schemata are admitted. The paper presents a process, model and algorithms to manage the lifecycle, refinement and dynamic combination of semantic mappings for these data and service description schemata that harness federation context and automated correspondence pattern recognition. The work is notable for its emphasis on maxmising the degree to which the generated executable mapping components can be reused by multiple mappings.

The second article, "Ontology-based Semantic Priority Scheduling for Multidomain Active Measurements," by Calyam et al., also proposes the use of semantic models, albeit for a very specific purpose-the development of an ontology-based semantic priority scheduling algorithm that prioritises measurement requests from multiple sources. The article addresses the deployment of openly accessible multidomain active measurement frameworks, such as perfSonar, which results in users competing for network measurement resources. The semantic priority scheduling algorithm prioritises measurement requests based on user roles, user sampling preferences, resource policies, and oversampling mitigation factors. The performance evaluation results presented, which are based on real-world measurement request parameters and multi-domain considerations, suggest that the proposed scheme outperforms previously proposed approaches, in particular significantly improving the satisfaction ratio among users.

The penultimate article, "An Iterative Approach to Trustable Systems Management Automation and Fault Handling," by McLarnon et al., notes that automated management systems are increasingly being used to automate common management tasks for large-scale distributed computing systems, but points out that they come with some disadvantages, especially when it is necessary to manage resources in external management domains. In particular, they argue that the abstraction that these systems create between system administrators and the managed resources mean that when an error occurs or a task fails it becomes significantly more difficult to identify the root cause. To counter this, the article proposes an approach to automated systems management in which the administrator is able to transition control of management tasks to an automation solution in stages. Furthermore, the authors describe how analyses of fault condition patterns can be used in future task iterations to automatically determine the completion state. It is clear that automated management systems whose operation can be governed by administrators in the 
manner described will be necessary to manage the increased complexity of federated network environments.

The final article, "End-to-End Resource Management for Federated Delivery of Multimedia Services," by Famaey et al., addresses how federation techniques can provide Quality-of-Service (QoS) aware delivery of streaming multimedia across the Internet. The article proposes an automated mechanism to set up end-to-end federations comprising a content provider, its customers and the intermediary network domains that connect them. Moreover, a federated resource reservation algorithm is presented, which facilitates identification of the optimal set of stakeholders and resources to include within such a federation. Another significant addition is the ability to harness intermediate storage sites to be included in the federation in order to increase the QoS provided to customers. As a whole, the framework offers a compelling argument for the benefits of introducing flexible federation capabilities into future networks.

\section{Conclusion}

In addition to the articles in this special issue, readers interested in management of federations may also wish to explore the articles published in the December 2013 special mini-issue of JNSM on Efficient Management of Loosely Cooperative Service Networks [1] and in the November 2013 special section of IEEE Communications Magazine on Monitoring and Troubleshooting Multi-domain Networks using Measurement Federations [2].

We would like to take the opportunity to thank the authors who submitted very high quality manuscripts to the special issue and who spent significant efforts to revise their manuscripts in line with reviewers' comments. We also would like to thank the anonymous reviewers who volunteered to review the manuscripts and who provided insightful and useful feedback to the authors. Finally, we would like to acknowledge the efforts of the JNSM editor-in-chief, Prof. Deep Medhi, who gave use the opportunity to organise this special issue and who gave us very good advice and guidance. We hope that readers will find the selected articles useful for their own work on topics relating to management of federations.

Acknowledgments Brendan Jennings and Kevin Feeney acknowledge the support of Science Foundation Ireland via the FAME strategic research cluster (Grant No. 08/SRC/I1403).

\section{References}

1. Special mini-issue: efficient management of loosely cooperative service networks. J. Netw. Syst. Manag. 21(4), 535-706 (2013)

2. Calyam, P., Dovrolis, C., Jörgenson, L., Kettimuthu, R., Tierney, B., Zurawski, J.: Monitoring and troubleshooting multi-domain networks using measurement federations [guest editorial]. IEEE Commun. Mag. 51(11), 53-54 (2013). doi:10.1109/MCOM.2013.6658652

3. Dobson, S., Denazis, S., Fernández, A., Gaïti, D., Gelenbe, E., Massacci, F., Nixon, P., Saffre, F., Schmidt, N., Zambonelli, F.: A survey of autonomic communications. ACM Trans. Auton. Adapt. Syst. 1(2), 223-259 (2006). doi:10.1145/1186778.1186782 
4. Feeney, K., Fleck II, J.J., Strassner, J., Won-Ki Hong, J., Choi, T., O’Sullivan, D., Pfeifer, T. (eds.): Combined Proceedings of 1st IFIP/IEEE Workshop on Managing Federations and Cooperative Management (ManFed.CoM 2011) and the 3rd IFIP/IEEE International Workshop on Management of the Future Internet (ManFI 2011), No. 14 in Multicon Lecture Notes. Multicon verlag, Berlin (2011)

5. Feeney, K., Jennings, B., Fleck II, J.: Managing federations and cooperative management: a report on ManFed.Com 2011. J. Netw. Syst. Manag. 20(1), 143-148 (2012). doi:10.1007/s10922-011-9214-0

6. Jennings, B., Brennan, R., Donnelly, W., Foley, S.N., Lewis, D., O'Sullivan, D., Strassner, J., van der Meer, S.: Challenges for federated, autonomic network management in the Future Internet. In: Proceedings of the 1st IFIP/IEEE International Workshop on Management of the Future Internet (ManFI 2009), pp. 87-92. IEEE (2009). doi:10.1109/INMW.2009.5195942

7. Jennings, B., van der Meer, S., Balasubramaniam, S., Botvich, D., Ó Foghlú, M., Donnelly, W., Strassner, J.: Towards autonomic management of communications networks. IEEE Commun. Mag. 45(10), 112-121 (2007). doi:10.1109/MCOM.2007.4342833

Brendan Jennings (http://brendanjennings.net) is the Director of Research with the Telecommunications Software \& Systems Group at Waterford Institute of Technology, Ireland. He was awarded B. Eng. and Ph.D. degrees by Dublin City University, Ireland in 1993 and 2001 respectively. During 2012 he was a visiting researcher at the Royal Institute of Technology (KTH) in Stockholm, Sweden and during 2014 he is a visiting researcher at $\mathrm{EMC}^{2}$ Research Europe in Cork, Ireland.

Kevin Feeney is a Research Fellow in the Knowledge and Data Engineering Group (KDEG), Trinity College Dublin (TCD), Ireland. His research focuses on distributed and decentralised management systems. He has spent the last 15 years modelling, designing and developing distributed systems, spread evenly across industry and academia, in areas spanning telecommunications and financial systems to consumer internet applications. He has published more than 30 papers on distributed management problems.

Joel J. Fleck II is Senior Standards Architect in the Global Technology Program of HP Labs and head of standards for HP Cloud Activities. His primary research and standards interests are adaptive management of distributed systems and policy based management. Prior to joining Hewlett-Packard, he spent 16 years at Bell Laboratories and Bell Communications Research researching distributed management systems, multimedia networks and automated test systems to validate digital communications systems. Joel graduated from the University of Michigan with a MS in Industrial and Operations Engineering and the University of Vermont with a B.S. in Computer Science. He is Distinguished Fellow of the TeleManagement Forum. 